\title{
POSITION-DEPENDENT MOTOR INNERVATION OF THE CHICK HINDLIMB FOLLOWING SERIAL AND PARALLEL DUPLICATIONS OF LIMB SEGMENTS ${ }^{1}$
}

\author{
VIRGINIA WHITELAW ${ }^{*, 2}$ and MARGARET HOLLYDAY $\ddagger^{3}$ \\ * Department of Biophysics and Theoretical Biology and $\ddagger$ Department of Pharmacological and Physiological Sciences, \\ The University of Chicago, Chicago, Illinois 60637
}

Received August 11, 1982; Revised December 27, 1982; Accepted January 17, 1983

\begin{abstract}
In this paper we report investigations of the innervation of duplicated limb segments to test whether the addition of limb segments along the proximodistal axons could stimulate the growth of appropriate motoneurons into double occurrences of these muscles. Our evidence indicates that it does not.

Using retrograde horseradish peroxidase nerve-tracing techniques and reconstructions of experimental limbs, we investigated the motor projection to parallel and serially duplicated legs. In all cases, host limb segments were normally innervated. In a control experiment involving a host thigh connected to a graft calf, the innervation of both segments was normal.

In serially duplicated limb segments, however, we found abnormal innervation. In limbs of the type thigh-thigh-calf-foot, the innervation of the second thigh was accomplished by calf motoneurons. In limbs consisting of thigh-calf-calf-foot, the duplicated calf was served by foot motoneurons. The general pattern was that muscles were innervated as a function of their position along the proximodistal axis, irrespective of their identity. In no limb were axons found distal to the third limb segment even before the period of normal cell death. Despite the mismatched innervation with regard to the thigh/calf/foot distinction, axons retained their characteristic selectivity for either dorsally or ventrally derived muscles.

The findings suggest that the projection of axons along the proximodistal axis of the limb is influenced by proximal growth cues associated with the formation of the limb plexus as well as by compctitive interactions in the distal limb tissue.
\end{abstract}

Patterns of neural activity are translated into functionally coordinated limb movements by means of a precise pattern of connections between motoneurons and muscles. The developmental mechanisms involved in organizing this motor projection can be studied by challenging the outgrowing axons with an altered periphery. The limb segment deletions discussed in the previous paper (Whitelaw and Hollyday, 1983a) provide evidence that both the motoneurons and the limb tissues are individuated such that motor axons respond selectively and appropriately to growth cues in the limb bud which lead

\footnotetext{
'This work was supported by National Institutes of Health Grant NS-14066 to M. H. V. W. was supported by United States Public Health Service Grants GM-07183, GM-07839, and NS-14066. We wish to thank Dr. Paul Grobstein for valuable discussions and for help in writing this paper.

${ }^{2}$ Present address: Bell Laboratories, Naperville, IL 60566.

${ }^{3}$ To whom correspondence should be sent at Department of Pharmacological and Physiological Sciences, University of Chicago, 947 East 58th Street, Chicago, IL 60637.
}

them into the vicinity of their normal targets. No claim is made, however, that pathway guidance cues are sufficient to pattern the entire motor projection. Indeed, we speculated that competition between axons normally traversing common pathways may also be important for normal limb innervation. 'The existence of multiple development mechanisms is suggested by experiments in which one or another apparently dominates (see Hollyday and Grobstein, 1981). For example, in chick embryos with supernumerary limbs, some normally thigh-serving motoneurons innervate calf muscles and vice versa (Hollyday et al., 1977; Hollyday, 1981; Lance-Jones and Landmesser, 1981b), although each maintains an ability to discriminate between dorsal and ventral muscle mass. In this situation, then, target choice is influenced by where motor axons enter the transplanted or host limb and by the fact that they choose their normal dorsally or ventrally destined pathways through it. Understanding the relevant difference between situations in which motoneurons innervate their usual target and those in which they 
innervate an abnormal target is central to understanding the development of the motor projection. Systematic behavior of motor axons under a defined set of experimental circumstances provides clues about the relative influence of various developmental mechanisms.

As shown in the previous paper (Whitelaw and Hollyday, 1983a), experiments on embryos with partial limbs demonstrate that motor axons selectively innervate thigh or calf muscles when the entire motor column projects to one leg segment. The pattern of selective innervation appears to result from axons responding differently to the growth cues in the truncated limb tissue such that only the appropriate axons penetrate the remaining muscles and form effective synapses; inappropriate motoneurons were excluded and they subsequently died. In this paper, we use serial and parallel duplications of limb segments to test whether the additional limb segments would be innervated by their appropriate motoneurons despite their novel positions.

\section{Materials and Methods}

All operations were done on White Leghorn chick embryos, staged according to the system of Hamburger and Hamilton (1951). Surgery was performed prior to nerve ingrowth into the limb bud, as described previously (Whitelaw and Hollyday, 1983a).

Surgery. Operations to make duplicated limbs were performed on stage (St) 20 to 22 embryos. The intent was to create limbs with extra limb segments (i.e., thigh, calf, and foot) attached to the ankle of the host limb. To do this, the distal tip (including the apical ectodermal ridge) of the host's right leg bud was removed. A donor limb bud was grafted to the distal portion of a truncated host limb bud. A schematic illustration of the operation is shown in Figure 1. In some cases, the grafted tissue was held in alignment with a fine tungsten needle for a period of 12 to $24 \mathrm{hr}$ until the graft had healed onto the

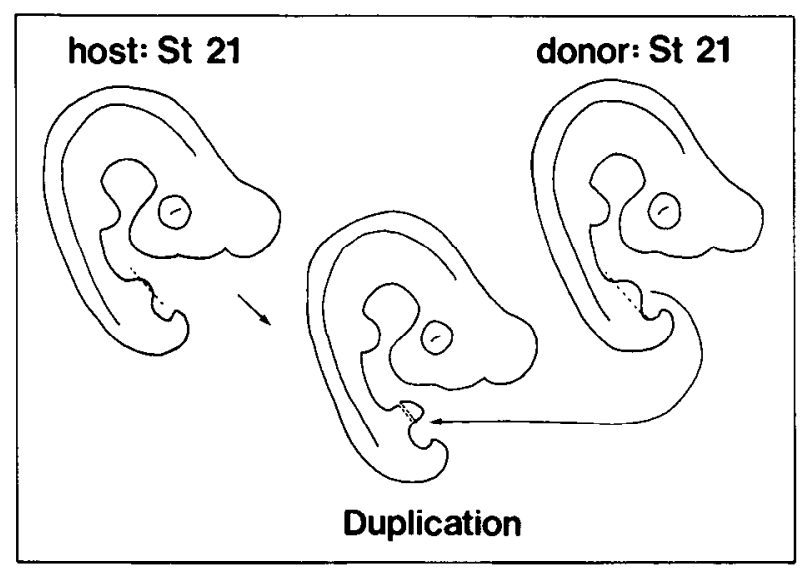

Figure 1. Schematic representation of limb segment duplication operation. Segment duplications were done by transferring a St 21 leg bud to a comparably staged host. The apical ectodermal ridge of the host was removed and the graft was held in place by the surrounding membranes. In general, the additional limb segments were attached serially to the host. In some cases, the graft healed in a dorsally displaced orientation relative to the host, producing a parallel duplication or forked limb. All operations were performed on the right leg bud. host. The pin was then removed. In most cases, however, a pin was not used, but rather excess fluid was simply wicked away in such a way that the membranes surrounding the graft tightened around it and held it in place.

A control set of operations was also performed in which approximately one-half of the host limb bud was excised and the distal half of a donor limb bud was transferred, so as to produce limbs having a host thigh with a graft calf and foot. Surgery was performed on approximately 170 embryos. Two-thirds of these animals (106) survived having successful duplications. Of these, approximately 60 animals gave positive histological results (see below).

Histological analysis. Motor pools serving duplicated muscles were mapped in St 36-38 embryos using intramuscular injections of horseradish peroxidase (HRP), as described previously (Whitelaw and Hollyday, 1983a). Injections were done either in ovo or in organ culture. The experimental limbs were carefully reconstructed, as before, to identify the musculature, the peripheral innervation pattern, and, where appropriate, the HRP injection site.

The peripheral nerve-branching patterns in younger experimental embryos were also studied using conventional histological techniques before and during the period of cell death to assess the extent to which they were altered by it. In addition to the hematoxylin and eosin staining method described in the previous paper, several embryos were studied using a silver stain (Ramon y Cajal and DeCastro, 1933). In both staining methods, the experimental right leg and normal left leg were left attached to the spinal cord and processed for paraffin sections. Sections were cut at $15 \mu \mathrm{m}$, transverse to the rostrocaudal axis of the spinal cord. Serial reconstructions of the peripheral branching patterns were made. The innervation of the manipulated limbs could be readily compared to what was seen in the normal left legs.

\section{Results}

\section{Characteristics of St 36-38 duplicated limbs}

Duplicated limbs were characterized by the identity and positions of their skeletal elements. Thigh and calf cartilage are distinctive, since the latter consists of two bones which run in parallel, the tibia and fibula, while the former only has one bone, the femur. Several types of duplicated limbs were made including the following configurations: thigh-calf-thigh-calf-foot (Fig. 2A), thighthigh-calf-foot, and thigh-calf-calf-foot (Fig. 2B). Although we attempted to make limbs with complete, duplicated segments, in reality some of the embryos had partially truncated limb segments. Embryos having truncated limb segments consisting of less than half a normalsized segment were discarded from the experimental series.

The majority of the limbs used in the motor pool study had only one segment serially duplicated, either the thigh or the calf. In some cases a graft segment ran alongside a host segment or was forked at a joint. These limbs were classified as parallel duplications (as distinct from serial duplications) and will be discussed separately.

Despite the additional limb segments, the total mass 

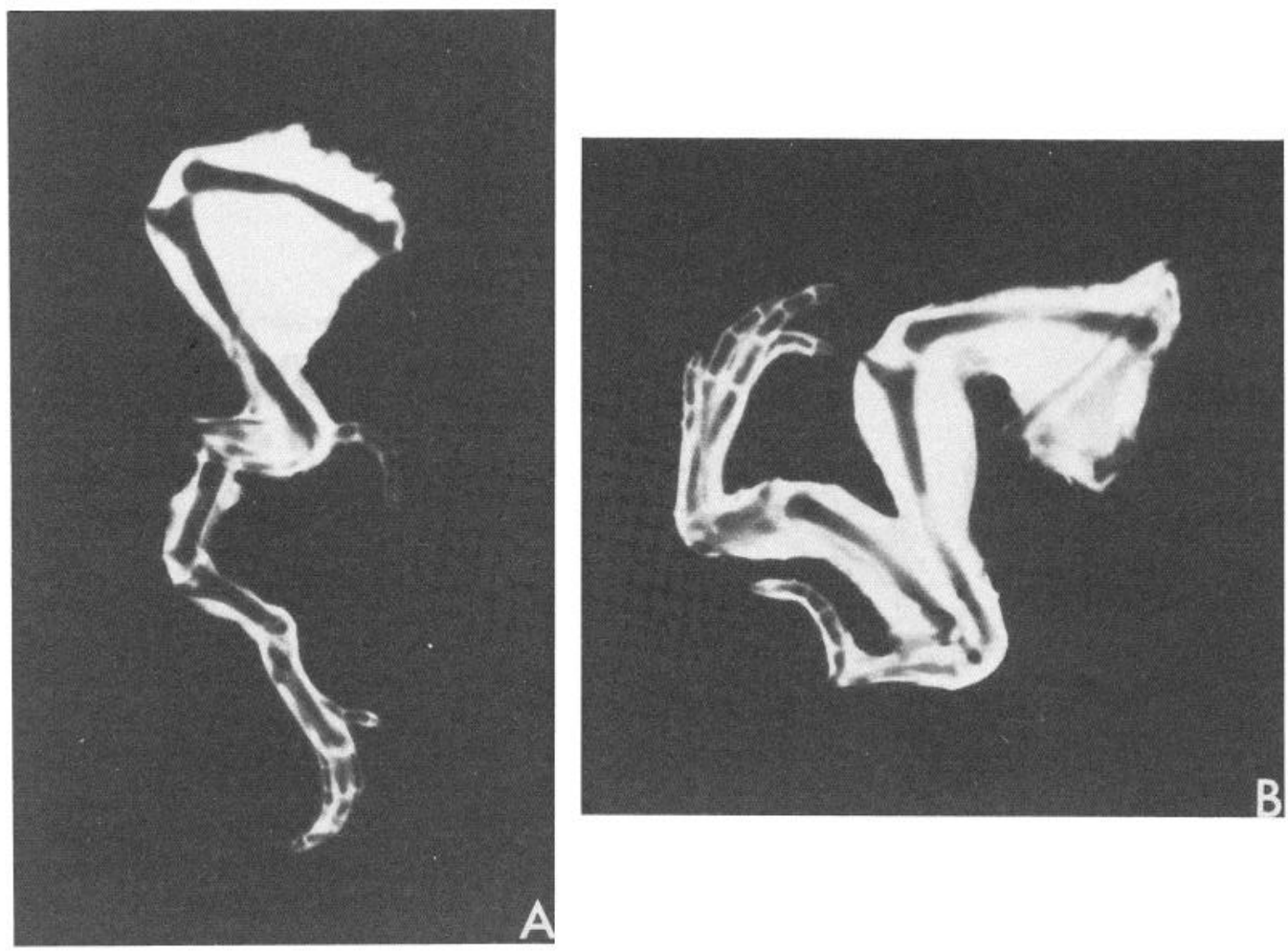

Figure 2. Whole mount serially duplicated limbs stained for cartilage. A, Duplication of the type thigh-calf-thigh-calf-foot, where the graft emerges from the distal end of the host calf. Noteworthy is the extensive muscle atrophy in the distal leg segments. $B$, Duplication of the type thigh-calf-calf-foot. There is one digit protruding from the host calf. All limbs are from St 36 embryos and are shown 5 times the actual size.

of duplicated limbs was not significantly greater than that of normal limbs. Indeed the mean mass of six duplicated limbs (at St 36) was found to be virtually the same as that of their normal contralateral counterparts, although the variance in their mass was greater (duplicated limb mean mass: $38.3, \sigma=4.2 \mathrm{mg}$; normal limb mean mass: $39.4, \sigma=2.9 \mathrm{mg}$ ). Consistent with these measurements was our observation that the size of the muscles was reduced in duplicated limbs, particularly in the distal, grafted segments, as evident in Figure 2 . The amount of musculature in duplicated limbs seemed to vary with a number of factors including the exact orientation of the graft, the extent of early vascularization, and the amount of eventual graft innervation-conditions which themselves were probably related. The best developed grafts were those which healed with proper axial alignment and received good vascularization soon after (within $12 \mathrm{hr}$ ) the time of operation.

Examination of histological sections revealed clear differences in the appearance of innervated and uninnervated muscles. The former had distinct grain patterns, while the latter had a homogeneous, amorphous appearance. Muscle innervation could be confirmed by distinct morphology of nerve entry into the muscle.

\section{$H R P$ injections of host limb segments}

HRP injections were made into muscles in the most proximal (host) thigh in embryos with serially duplicated thighs or calves. In each embryo, labeled cells were found in the appropriate thigh motor pools. Four animals which had duplicated thighs received injections into the most proximal occurrence of a specific thigh muscle. Both dorsal and ventral thigh muscles were sampled; successful injections were made into the femorotibialis (FT), iliofibularis (IFB), adductor (AD), and ischioflexorius. In all cases, label was confined to the muscle's normal motor pool.

Similarly, when the host limb was composed of both a thigh and a calf, HRP injections into muscles of the most proximal (host) calf labeled the appropriate calf motor pools. Six calf-duplicated embryos received injections into either dorsal or ventral host calf muscles. In all of these animals, the host calf was clearly proximal to the grafted tissue. Injections of ventral calf muscles appropriately labeled the caudomedial cluster of motoneurons; dorsal injections resulted in labeling of the normal intermediate calf pools. In the most heavily injected of these cases, there were virtually no unlabeled cells evident in the motor pools for either the peroneus longus (PL) or the tibialis anterior (TA), which are the two largest muscles of the dorsal calf. Moreover, the rostrocaudal extent of the pools appeared normal.

\section{HRP injections of grafted control (unduplicated) limbs}

A control series of operations was done in order to determine that grafted tissue per se was not avoided or aberrantly innervated by the nerves. In this set of experiments, legs were produced consisting of a host thigh and a graft calf. HRP injections of the calf tissue of eight such limbs resulted in label in normal calf motor pools. Injections of dorsal muscles appropriately labeled motoneurons in caudo-intermediate positions in the motor 
column; ventral muscle injections resulted in labeled cells in caudomedial positions. There were, on average, more than 250 labeled cells per case, which is comparable to the number of cells which label following similar injections into normal calves.

\section{HRP injections of serially duplicated thighs}

Second limb segments. The appearance of the lateral motor column (LMC) on the side of the duplicated limb segments did not differ appreciably from that of the control side. Neither an increase nor a decrease in cell number was apparent, but we did not make cell counts to study this point in detail.

The position of duplicated limbs along the proximodistal axis was a relevant factor in determining their innervation. Therefore, results are grouped according to where the injected limb segments occurred along this axis, as well as by the identity of the injected segments.

HRP injections into muscles of duplicated thighs did not label normal thigh motor pools. Five embryos which had duplicated limbs of the type thigh-thigh-calf-foot received successful HRP injections into one or more of the muscles of the second (graft) thigh. Labeled motoneurons were consistently found in positions which normally supply calf muscles.

The distribution of labeled cells from all five cases in which a second segment-duplicated thigh was injected are summarized in Table I. In two embryos, both dorsal and ventral muscles were injected together. In three others, individual muscles were injected (see below). This table is structured so as to represent the motor column on a rectangular grid. The rostrocaudal axis is shown horizontally and is demarcated by ventral root numbers. The mediolateral axis, shown vertically, is divided into thirds: "M," "I," and "L" designate "medial," "intermediate," and "lateral" positions, respectively. (Intermediate motor pools are those which have both medial and lateral neighboring pools.) The averaged percentages of labeled cells in each position are indicated, and the normal positions of motoneurons serving the injected muscles are indicated with heavy lines.

What is clear in Table $I$ is that there are virtually no labeled cells in any thigh motor pool position. In no case is there evidence of thigh motor axons innervating any muscle distal to the first thigh. The grafted thigh was innervated instead by motoneurons which normally supply calf muscles.

A selective mismatch of motor pools innervating the second thigh was consistently observed in the three animals that received specific injections of either a dorsal or a ventral thigh muscle. The motor pools supplying two muscles normally innervated from the crural plexus (AD and FT) and two muscles from the sciatic plexus (posterior iliotibialis (PIT) and IFB) were mapped. When the ventrally derived muscle, $A D$ was injected, medially lying motoneurons were labeled in caudal spinal segments. Motoneurons in these positions normally innervate ventrally derived calf muscles. When the dorsally derived muscles, FT, PIT, or IFB were injected, label was found in the caudo-intermediate position. In normal embryos, these motoneurons supply dorsally derived calf muscles.

Figure 3 shows a sample reconstruction of the labeling pattern following an injection into the PIT and IFB of the second, distal thigh. The normal motor pools for these muscles (shown in shading) are in a far lateral position. In this case, the vast majority of labeled cells were in the caudo-intermediate clusters which normally serve calf muscles TA and PL. None of the label was found in the normal PIT or IFB motor pools. However, all four muscles (PIT, IFB, PL, and TA) are dorsal mass derivatives. In this sense, the mismatch of motor pools is still partially appropriate. The innervation was incorrect in that it was accomplished by calf motor pools, but it was correct with respect to the dorsal/ventral muscle mass distinction.

The finding of a selective mismatch of motor pools serving duplicated thigh muscles is entirely consistent with what is seen in supernumerary limb innervation (Hollyday, 1981). In both manipulations, the thigh/calf distinction in motor pools can be overriden, but the dorsal/ventral distinction is respected.

Third limb segments. When HRP injections were made into duplicated thighs in the position of the third limb segment (i.e., limbs having the configuration thighcalf-thigh-calf-foot), there was rarely label in the motor column. The segments generally appeared very atrophic (see, for example, Fig. 2A) and as will be discussed later, reconstructions of the limbs revealed few, if any nerves in the third segment thighs.

In only two cases was a dorsal distal (third segment)

TABLE I

Distribution and averaged percentages of labeled cells following dorsal and/or ventral HRP injections of duplicated thighs (second limb segment) ${ }^{a}$

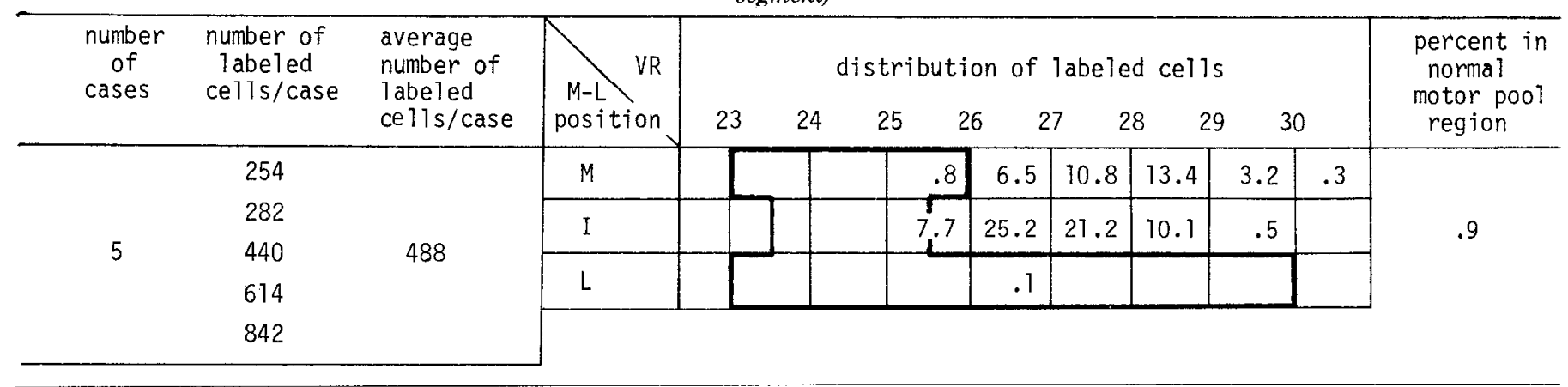

\footnotetext{
${ }^{a}$ Heavy lines indicate the position of motor pools innervating thigh muscles in normal animals. M, medial; I, intermediate; L, lateral.
} 


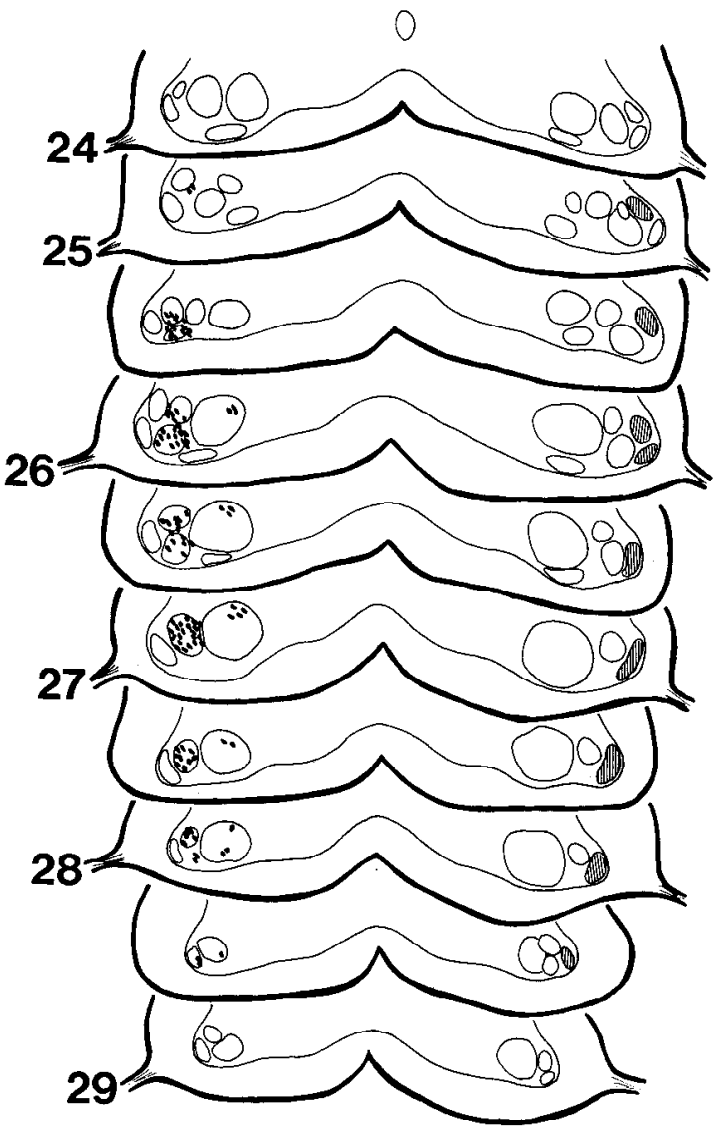

Figure 3. Reconstruction of the distribution of labeled cells following an HRP injection of the PIT and IFB in a duplicated (second segment) thigh. The normal motor pools for these muscles are shaded on the right-hand side of the spinal cord (as viewed by the reader, from Hollyday, 1980). The labeled cells are not in the corresponding position on the experimental side. Rather, they are in the caudo-intermediate pools which normally serve dorsal calf muscles. There are a few labeled cells in the medial calf motor pools (probably the result of HRP leakage), but there are no labeled cells in thigh motor pools.

thigh successfully injected. The resultant label differed both quantitatively and qualitatively from what was seen following more proximal injections. First, the average number of labeled cells in these two cases was far less than in the five cases listed in Table I (an average of 13 labeled cells per case as opposed to an average of 488 labeled cells per case in the other five cases). Second, the labeled cells were in a slightly different position than what was seen earlier; namely, they were situated at the lateral peripheral border of the medial cluster. It is difficult to draw conclusions from only two very sparsely labeled cases. However, the labeling is in the same position in which labeled cells are often found following dorsal muscle injections of duplicated calves, as discussed in the following section. It is the normal position of a motor pool which serves a dorsally derived, intrinsic foot muscle.

\section{HRP injections of serially duplicated calves}

Third limb segments. Serially duplicated calves were either the third or fourth leg segment along the proxi- modistal axis. HRP injections into the most distal duplicated calf often resulted in no labeled cells in the LMC. This was largely, if not entirely due to the generally poor innervation of the distal calf. As will be discussed later, serial reconstructions of the innervation of the duplicated limbs revealed few, if any, nerves in the distal calf. In only $25 \%$ ( 6 of 26 ) of the distal calf injections was any label found in the spinal cord, as compared with the roughly $90 \%$ "success rate" (14 of 16) following injections of host or graft calves in control animals. In those cases in which there was label following injections of the second calf, the duplications were of the type: thigh-calf-calffoot; that is, the grafted calf was the third distal segment, as in Figure $2 B$. The resultant label was generally sparse and located on the periphery of the medial cluster.

Figure 4 shows a sample reconstruction of the labeling pattern following distal ventral calf injection. This animal had a duplicated calf, but only a host thigh. The label, as shown in Figure 4, is confined to a far caudomedial position in the medial cluster. It is difficult to make a strong case for naming motor pools within the medial cluster as there is considerable overlap. However, the

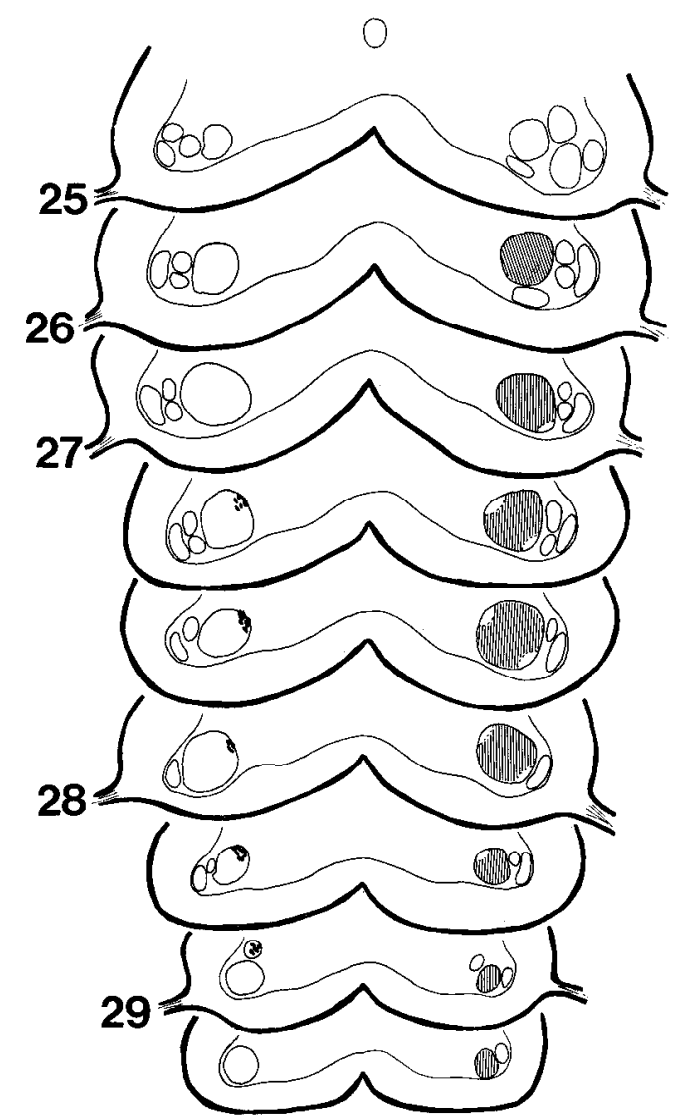

Figure 4. Reconstruction of the distribution of labeled cells following an HRP injection of ventral calf muscles in a duplicated (third segment) calf. The normal motor pools for the muscles injected are shaded on one side of the spinal cord (from Hollyday, 1980). The labeled cells are not in a corresponding position on the experimental side. Instead, they are in a far peripheral position within the medial cluster in a region which normally serves intrinsic foot muscles. As seen in this case, generally few motoneurons labeled following injections of third limb segments. 
position of the labeled cells exactly corresponds to the normal position of the motor pool serving the flexor hallucis brevis (FHB) (see Hollyday, 1980), which is one of the few muscles intrinsic to the foot. Extensor hallucis brevis (EHB) is the other major intrinsic foot muscle. EHB is dorsally derived and its motor pool is on the lateral border of the medial cluster.

In all six embryos in which there was a successful HRP injection of the most distal serial calf, label was found at the periphery of the medial cluster. Ventral muscle injections resulted in label roughly corresponding to the FHB motor pool; dorsal muscle injections labeled a region roughly corresponding to the EHB motor pool. Thus, a characteristic preference on the part of motoneurons for either dorsal or ventral muscles is again evident, despite the apparent mismatch of foot motor pools serving calf muscles.

These findings are completely analogous to the results of duplicated thigh injections in which calf motoneurons innervate the second thigh, but with a characteristic preference for dorsal or ventral muscles. All six cases in which labeled cells were found following HRP injections of duplicated third segment calves are summarized in Table II. As in the previous table, the motor column is represented on a rectangular grid. The identification of mediolateral positions, however, is somewhat different. There are two positional designations distinguishing the periphery of the medial cluster from its center. "Peripheral-medial" (P-M) and "peripheral-intermediate" (P-I) refer to opposite sides of the medial cluster. At caudal spinal levels, these are the positions of motor pools which normally serve the intrinsic foot muscles. The ventral muscle, FHB, is served by a peripheralmedial crescent of motoneurons; the dorsal muscle, EHB, is served by a peripheral-intermediate cluster. Caudal motoneurons in the medial (M) and intermediate (I) positions normally serve ventral and dorsal calf muscle, respectively (shown with heavy lines in Table II). (The lateral designation which corresponds to thigh motor pools has been omitted in this table.) Results from both dorsal and ventral muscle injections are combined. As is evident in Table II, labeled motoneurons were found almost exclusively on the edges of the most medial motoneuron cluster, which is where foot motor pools are normally found; there are virtually no labeled cells in the normal calf motor pool regions.

A second distinguishing feature of the HRP label following distal calf injections is its scarcity. The amount of label in these six peripherally labeling cases is roughly an order of magnitude less than that obtained following injections of more proximal segments. (The amount of HRP injected into these distal segments was comparable to, if not greater than, the size of the injections into the more proximal leg segments.) Following injections of the second limb segment, whether it was a grafted (but not duplicated) calf or a grafted thigh, there were on average more than 350 labeled cells per case. Following injections of the distal calf, a third leg segment, an average of 24 labeled cells per case was found. Interestingly, this latter figure is comparable to what was obtained in the two grafted thigh injections described in the previous section in which the second thigh was preceded proximally by both a host thigh and by a large host calf and thus was properly a third distal leg segment. Furthermore, it may be recalled that the label in these two thigh-injected cases was also peripheral in the medial cluster.

Taken together, the results from both duplicated thigh and calf experiments suggest that motor innervation in duplicated segments is dependent upon where those segments occur in the limb (i.e., whether they are a second or third limb segment along the proximodistal axis). It seems to have little to do with whether the segment being innervated is a thigh or a calf.

\section{HRP injections of parallel duplications and twinned limbs}

In addition to serial duplications of limb segments of the kind described above, a small number of more abnormal limbs resulted from embryonic manipulations. These involved cases in which two duplicated segments lay side by side. In some cases the duplicated segments were fused with one another. In others ("twinned" or "forked" limbs), the two segments met at the proximal joint and diverged from one another at that point.

Nine such animals having a parallel duplication or a twinned segment in the position of the second segment were studied. Irrespective of whether the duplicated tissue was of thigh or calf origin, and whether the duplica-

TABLE II

Distribution and averaged percentages of labeled cells following dorsal or ventral HRP injections of duplicated calves (third limb segment) ${ }^{a}$

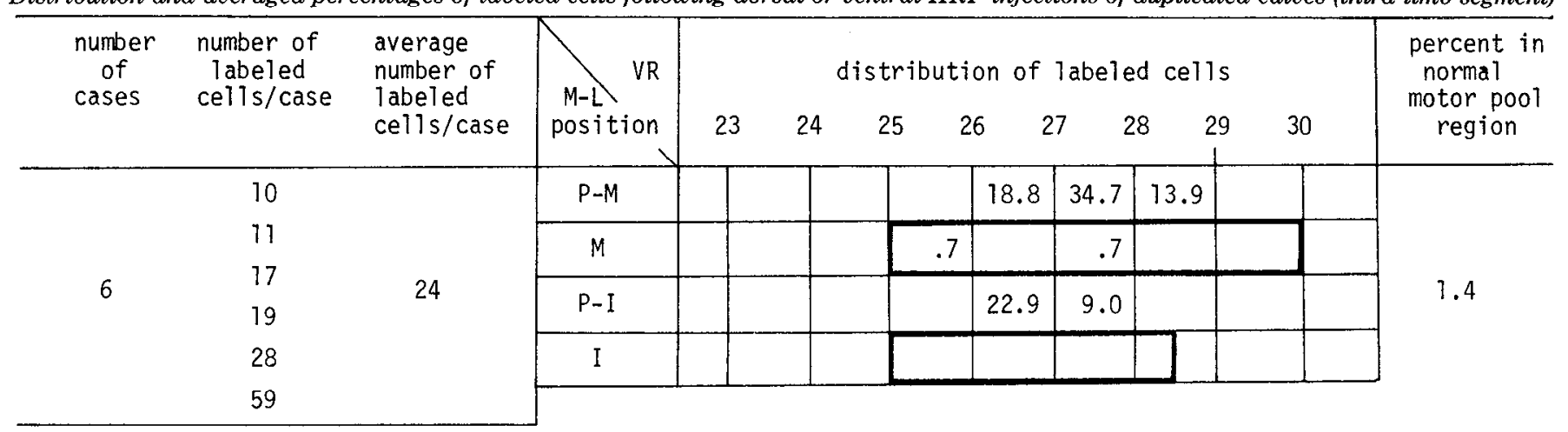

\footnotetext{
${ }^{a}$ Heavy lines indicate the positions of motor pools innervating calf muscles in normal animals. P, peripheral; M, medial; I, intermediate.
} 
tion was of the fused or forked type, calf motor pools were always labeled when the duplicated tissue was injected with HRP. In six such embryos, dorsal muscles were injected and labeled cells were found in caudointermediate positions, characteristic of motoneurons going to dorsally derived muscles. In three other cases in which ventral muscle mass was injected, medially lying cells were labeled. Hence, in these nine cases, the label was appropriate with regard to the dorsal/ventral muscle mass distinction, although in some instances the label was inappropriate, with respect to the thigh/calf discrimination.

In a single embryo having a duplicated calf attached to the lateral surface of the host calf, the usual innervation pattern of dorsally and ventrally derived muscles was not found. Labeled motoneurons were found only in caudo-intermediate positions appropriate for dorsal calf muscles and not in any medially lying motoneurons which normally innervate ventral calf. In this embryo, a large, intentionally diffuse injection of HRP had been made into both dorsal and ventral muscle in the grafted calf. Reconstruction of the nerve supply to this limb revealed that it differed from the other duplicated limbs studied in that a branch from a single nerve, the peroneal nerve (which normally supplies only dorsally derived calf muscles), entered the graft. All of the other duplicated limbs studied were supplied by branches from both the peroneal and tibial nerves, if they received any innervation at all (see below).

Following injections of parallel or twinned limb segments, there were, on average, more than 200 labeled cells per case. This quantity is comparable to that obtained following other injections of second limb segments and is roughly an order of magnitude greater than that seen following third segment injections as described below. In addition, the distribution of label following these second segment injections was different from that found following more distal injections in that it was not confined to the periphery of the medial cluster of motoneurons.

Several animals having limbs with twinned feet were produced, two cases of which were successfully injected with HRP. The resultant label was located peripherally in the medial cluster, as is characteristic of motor pools serving intrinsic foot muscles. In one case, an injection was made into the ventral muscle of one of the feet. Label was found at the far medial periphery of the medial cluster. In the other case, one of the feet received injections into both dorsal and ventral muscles. Label resulted at both peripheral borders (lateral and medial) of the medial cluster. In both cases, the number of labeled cells was low (average, 27.5 labeled cells per case). This is exactly the labeling pattern found following injections of normal feet, indicating the normalcy of foot innervation in twinned limbs. Moreover, these are examples in which the parallel or lwinned limb segments occurred as third segments along the proximodistal axis. The amount and position of label are entirely consistent with what was obtained following injections of the third leg segment in serially duplicated limbs.

In summary, then, the motoneurons serving the second limb segment are in the same positions in the motor column, irrespective of whether that segment is a host calf, a donor thigh, a donor calf, a parallel duplication, or a forked limb. The motor pools serving the third limb segment are in the same positions whether that segment is a normal foot, a twinned foot, or a duplicated thigh or calf.

\section{Nerve reconstructions from duplicated limbs}

The interpretation of the motor pool studies described above depends in part on information about the nerve pathways supplying muscles in the duplicated segments. Serial reconstructions of many of the St 36-38 duplicated limbs revealed an interesting mixture of normalcy and novelty in the peripheral nerve-branching patterns. The peripheral patterns were novel inasmuch as some of the limbs contained unusually positioned cartilage or muscle at the host-graft juncture that had to be bypassed or innervated.

All of the duplicated limbs, whether of the serial or parallel type, had normally innervated host thighs in the position of the first segment. This is consistent with our findings that host thigh muscles were innervated by their normal motor pools. Nerves emerging from the crural plexus were exclusively distributed to anterior host thigh muscles. Individual nerves from the sciatic plexus were distributed to posterior thigh muscles. If nerves entered the grafted segments at all, they could be identified as the peroneal and tibial nerves, by virtue of their characteristic positions in the host thigh. In serially duplicated thighs or calves, the peroneal nerve supplied dorsal muscles; the tibial nerve innervated ventral muscles. Once into the grafted tissue, the nerve-branching patterns were entirely appropriate for the particular limb segment. These findings are similar to those of Lewis (1978) on serially duplicated segments in the chick wing, and demonstrate the ability of axons to expand into the available pathways under these experimental conditions. In parallel duplications and forked limbs the innervation was typically from branches of both the peroneal and tibial nerves. The fact that in many serially duplicated legs, both the peroneal and tibial nerves entered the graft is not to imply that they innervated all of it. In many duplications, the nerves were found in the proximal graft tissue, but not in the distal segments.

The size of the peroneal and tibial nerves decreased with each segment along the proximodistal axis of the limb as motor axons peeled away to innervate the local muscles. Only very small nerve bundles were evident in the third leg segments. In no leg which had four or five limb segments (i.e., thigh-calf-thigh-calf-foot) were any nerves evident beyond the third limb segment. In other words, there was no expansion of motor innervation throughout the serially duplicated limb.

This last observation is important, as it accounts for the fact that numerous HRP injections of duplicated calves which were fourth leg segments resulted in no motor pool labeling. It may also be part of the explanation for the near-equal mass of duplicated and normal limbs. By St 36-38, uninnervated leg segments showed dramatic muscle atrophy. In some cases, little more than the skeletal elements remained. The fact that even the longest legs had only three innervated segments-and the third segment was sparsely innervated, at thataccounts for the extensive muscle atrophy observed in distal limb segments and thus in part for the fact that 
the mass of duplicated limbs did not scale with the number of segments.

In the limbs in which no nerves were found in the duplicated segments, the graft tissue had generally healed at an odd angle relative to the host, or there was an odd cartilage formation at the host/graft interface which apparently blocked passage of the nerve into the graft. In the limbs where the nerves innervated the host limb segments only, the muscles of the grafted segments showed considerable atrophy by St 36 , evident not only by their much-reduced size, but also by their degenerate appearance.

The absence of nerves beyond a certain proximodistal level in the adult-like duplicated limbs raised the question of whether, during development, the distal tissue was ever penetrated by motor axons. To investigate this question, silver-stained as well as hematoxylin-and-eosinstained serial sections of duplicated limbs were examined between St 28 and 34. (We did not study the early innervation patterns of serially duplicated limbs using orthograde transport of HRP because in our experience it is difficult to obtain labeled nerves beyond the second limb segment.) The period of natural cell death begins around St 28 and is roughly half over by St 34 (Hamburger, 1975). Axons normally reach the limb tip around St 29. Slightly later stages were examined to allow for the possibility that axons take longer to reach the tips of longer limbs.

Despite the fact that there are nearly twice as many motor axons in the periphery before cell death as there are after cell death, it was found that the gross features of their branching patterns did not change appreciably over this period. Axons from the crural plexus terminated in the first limb segment, those from the sciatic plexus primarily terminated in the first and second limb segments. Even near the beginning of the cell death period, there was little or no innervation of distal leg segments. At best, only small nerves were found in third limb segments. No nerves were in further distal segments. This finding before and during the cell death period is consistent with what is seen in post-cell death embryos.

\section{Discussion}

Selective mismatch. Except for the muscles of the host thigh, muscles in serially duplicated limbs generally did not receive innervation from their normal motor pools. Duplicated thighs were served by calf motor pools, duplicated calves were supplied by presumptive foot motor pools, and many muscles, particularly those lying distally in duplicated segments, received no innervation at all. Motor axons were not stimulated to growth farther distally than they normally do by the presence of additional limb tissue. The innervation of a given limb segment depended on its position along the proximodistal axis and not on its identity.

The overall pattern of innervation in this experimental situation seemed to be determined by the tendency of motoneurons to terminate in a particular location (i.e., in the first, second, or third limb segment) rather than at a particular muscle. However, despite the often observed mismatch of motor pools serving the second or third limb segment, those pools served muscles derived from the same embryonic muscles mass as were their normal targets. Intermediate-lying motor pools which would normally serve dorsal calf muscles, for example, innervated dorsal thigh muscles when the latter were in the second limb segment. This sort of mismatch is analogous to what is seen in supernumerary limbs. When the motor column serves two limbs on one side, calf motor pools can innervate thigh muscles (and vice versa for thigh motor pools), but they do so with a characteristic selectivity for either dorsally or ventrally derived muscles (Hollyday et al., 1977; Hollyday, 1981). Hence, while motoneurons did not generally terminate at their normal muscle in the duplicated limbs, they appear to have distinguished normally between dorsal and ventral muscles or the pathways that lead to them.

Comparison with partial deletion findings. There is a significant difference between the present results from duplications and those in the case of partial deletions described in the previous paper (Whitelaw and Hollyday, 1983a). The implication from the duplications is that segment position in the limb is central in determining its innervation, whereas the deletions suggest that if different limb segments are put in the position of the first limb segment, they are innervated in accord with the identity of that segment. In the partially deleted limbs, the remaining segment was a first limb segment, yet whether it was innervated by thigh or calf motor pools depended on whether it was a thigh or a calf. In the duplications, innervation of the second and third limb segments had little to do with what those segments were.

A difference between duplicated and partially deleted limbs that may be relevant to understanding their respective innervation patterns is that in deletions there is a vast excess of motoneurons relative to muscles, whereas in duplications this is not the case. In deletions, both thigh and calf motor axons are available to innervate the remaining segment. In that situation, only the appropriate motoneurons made effective synapses with the available muscle precursor tissue; the target-deprived motoneurons were excluded and died. As discussed in the preceding paper, we think it is likely that competitive interactions between the appropriate and target-deprived motoneurons are involved in excluding foreign motoneurons in partial hindlimbs. In embryos with duplicated hindlimbs, on the other hand, thigh motor axons are spent in the first limb segment, leaving only calf and foot motor axons to innervate the rest of the limb. In this situation, calf motoneurons can apparently innervate thigh muscles, and foot motoneurons can innervate the calf. The partial deletions provide evidence for thigh/calf selectivity. The duplications show this selectivity is not "all or nothing."

Cues for axonal guidance and growth. The pre-cell death peripheral nerve patterns indicate that axons not innervating the thigh do not explore the entire limb. Most of the axons go no farther than the second limb segment; none go farther than the third limb segment. Hence, our observations suggest that motor axons are subject to some kind of control on their proximodistal growth.

Such cues may be associated with the formation of the limb plexuses. We have found that axons associated with particular nerves grow for distances in the limb characteristic of those particular nerves, irrespective of the 
presence of additional appropriate targets or the identity of the distal tissue. It would seem that for motor axons to reach distal limb segments, it is sufficient for them to join either the peroneal or tibial nerves, which are the exclusive pathways for motor axons projecting distal to the thigh. Once having made that choice, the majority of axons in those nerves project to the second limb segment; a small number continue to grow to innervate the third limb segment.

What determines which motoneurons innervate the second segment and which go on to the third is not entirely clear. A hint comes from the observation that in normal animals, the innervation of the third segment is from a small number of motoneurons located at the medial and lateral borders of the medial motoneuron cluster, while the majority of the neurons centrally located in that cluster project to the second segment. We observed this same pattern in duplicated hindlimbs, even when the third segment was not a foot. Interestingly, in the case of supernumerary limbs, where normally thighdestined motoneurons innervated the second and third segment of a grafted limb, a similar center/perimeter subdivision within the neuron cluster was observed (see Figs. 10 and 17 of Hollyday, 1981). This suggests that the center/perimeter pattern is not an intrinsic or highly specified one, but rather, one which results from processes occurring in the limb and hence one which presumably involves interaction with the limb tissue. One possibility is that in the formation of the sciatic plexus, there is a sorting process such that axons from motoneurons at the perimeter of the group of neurons forming the tibial and peroneal nerves become located in a part of the nerve which predisposes them to grow to the most distal (third) segment, while the axons of more central motoneurons are sorted into positions in the nerve predisposing them to innervate the second segment. Another possibility is that motor axons from these perimeter motoneurons are at a competitive disadvantage in forming synapses in the second limb segment.

Limitations on graft innervation. In more than half of the duplicated limbs studied either before or after cell death, no nerves were found in the grafted tissue. This was particularly the case when the graft had healed in an abnormal alignment (e.g., dorsal to host limb) or when the graft was proximally preceded by a lot of host muscle. This frequent absence of innervation could have resulted from a lack of compatibility between motoneurons and grafted muscle, or from a lack of access to it. The former explanation seems implausible in view of the success with which grafted tissue was innervated in other manipulations. For example, in all cases in which donor calves were grafted onto host thighs, they were successfully and appropriately innervated. Hence it is likely that limitations on motor axon mobility prevented graft innervation in many cases. It seems more likely that if the grafted tissue (or pathways into it) did not lie within a prescribed neighborhood of the normal neural pathways, the nerves bypassed the graft, unaware of its presence.

Although nerves could not be induced to grow farther distally than normal in search of muscle targets, they could be induced to branch. In this sense, serial and parallel duplications provided different innervation contexts. Parallel duplications or forked limbs offered branch points to the nerves, and the nerves split appropriately to innervate either the dorsal or ventral muscles of each of the duplicated segments. The findings of Bennett et al. (1979) suggest that under these circumstances, homologous muscles are probably innervated by motoneurons located in similar parts of the spinal cord; this was not, however, explicitly tested in our animals. Serial duplications, on the other hand, challenged the nerves with a longer limb to innervate and the nerves clearly failed to do so. It is not clear in parallel or twinned limbs whether axons themselves branched at the bifurcated pathways, or whether the nerve bundle as a whole split as a result of each axon making an exclusive decision as to which pathway to take.

Comparison with early innervation studies. If each axon projects to only one of the two limb segments in twinned limbs, it would be entirely in keeping with what they do in other manipulated situations and during normal development. Studies on early innervation patterns suggest that as soon as motor axons grow into the limb, they grow to the region where their target muscle will eventually differentiate (Landmesser, 1978; Lance-Jones and Landmesser, 1981a). The relative accuracy of early innervation is incompatible with the idea that axons explore the entire limb in search of suitable targets. If, for example, thigh motor axons explored the calf and foot segments in search of compatible targets, the early innervation pattern would look far from normal. What duplication studies add to what is known about early innervation is that limb segments evidently cannot attract axons; they cannot make their thigh-like or calf-like presence known at a distance. As a result, axons behave in duplicated limbs much as they do in normal limbs: a certain fraction of axons grows no further than the first limb segment: another fraction progresses no further than the second, and a small fraction grows to the third.

In agreement with the early innervation studies of normal limbs, the pre-cell death innervation of duplicated limbs was substantially the same as what was seen in older embryos. Reconstructions of the nerves in duplicated limbs revealed that before and during cell death, the nerves from the crural plexus projected only as far as the host thigh (i.e., the first limb segment). Nerves from the sciatic plexus branched appropriately in the host thigh, and diverged into its tibial and peroneal subsets, which themselves branched in the second limb segment. In the duplicated grafts, by the level of the third distal leg segment, at best only small nerves could be found. No nerves were observed at farther distal levels. The fact that this pattern was not altered appreciably throughout the period of cell death supports the idea that cell death is not a process by which the adult innervation pattern is sculpted from grossly erroneous beginnings (see also Landmesser and Morris, 1975; Landmesser, 1978; Oppenheim, 1981).

The first paper in this series (Whitelaw and Hollyday, 1983a) reported findings indicating that motoneurons are individuated with respect to their ability to respond to cues in the limb bud. The observations reported in this paper indicate that pathway choices made at far proximal levels in the limb bud are important for deploying axons to particular limb segments along the proximodistal axis of the limb bud. We also suggest that interactions among 
axons growing along common pathways are important for patterning projections to distal limb muscles, as for example, to the calf and foot. In the following paper (Whitelaw and Hollyday, 1983b) we report some further evidence for the importance of peripheral growth cues and consider how the various factors which have been shown to influence projection patterns may interact in normal development.

\section{References}

Bennett, M. R., R. Iindeman, and A. G. Pettigrew (1979) Segment innervation of the chick forelimb following embryonic manipulation. J. Embryol. Exp. Morphol. 54: 141-154.

Hamburger, V. (1975) Cell death in the development of the lateral motor column of the chick embryo. J. Comp. Neurol. 160: 535-546.

Hamburger, V., and H. Hamilton (1951) A series of normal stages in the development of the chick embryo. J. Morphol. 88: 49-92.

Hollyday, M. (1980) Organization of motor pools in chick lumbar lateral motor column. J. Comp. Neurol. 194: 143-170.

Hollyday, M. (1981) Rules of motor innervation in chick embryos with supernumerary limbs. J. Comp. Neurol. 202: 439465.

Hollyday, M., and P. Grobstein (1981) Of limbs and eyes and neuronal connectivity. In Studies in Development Neurobiology. Essays in Honor of Viktor Hamburger, W. M. Cowen, ed., pp. 188-217, Oxford University Press, New York.

Hollyday, M., V. Hamburger, and J. M. G. Farris (1977) Localization of motor neuron pools supplying identified muscles in normal and supernumerary legs of chick embryos. Proc. Natl. Acad. Sci. U. S. A. 74: 3582-3586.

Lance-Jones, C., and L. Landmesser (1981a) Pathway selection by chick lumbosacral motoneurons during normal development. Proc. R. Soc. Lond. (Biol.) 260: 1-18.

Lance-Jones, C., and L. Landmesser (1981b) Pathway selection by embryonic chick motoneurons in an experimentally altered environment. Proc. R. Soc. Lond. (Biol.) 260: 19-52.

Landmesser, L. (1978) The development of motor projection patterns in the chick hindlimb. J. Physiol. (Lond.) 284: 391414.

Landmesser, L., and D. G. Morris (1975) The development of functional innervation in the hindlimb of the chick embryo. J. Physiol. (Lond.) 249: 301-326.

Lewis, J. (1978) Pathways of axons in the developing chick wing: Evidence against chemo-specific guidance. Zoon 6: 175179

Oppenheim, R. W. (1981) Cell death of motoneurons in the chick embryo spinal cord. V. Evidence on the role of cell death and neuromuscular function in the formation of specific peripheral connections. J. Neurosci. 1: 141-151.

Ramon y Cajal, S., and F. DeCastro (1933) Elementos de Tecnica Micrografica del Sistema Nervioso. Tipografica Artista, Madrid.

Whitelaw, V., and M. Hollyday (1983a) Thigh and calf discrimination in the motor innervation of the chick hindlimb following deletions of limb segments. J. Neurosci. 3: 1199-1215.

Whitelaw, V., and M. Hollyday (1983b) Neural pathway constraints in the motor innervation of the chick hindlimb following dorsoventral rotations of distal limb segments. J. Neurosci. 3: 1226-1233. 